\title{
UNA NUEVA ESPECIE DE PASSIFLORA SECCIÓN DECALOBA (PASSIFLORACEAE) DE PERÚ
}

\author{
Boris Esquerre-Ibañez
}

\begin{abstract}
Laboratorio General de Biotecnología, Universidad Nacional Pedro Ruiz Gallo, Lambayeque, Perú; kamijo002@gmail.com (autor corresponsal).
\end{abstract}

\begin{abstract}
Esquerre-Ibañez, B. 2019. A new species of Passiflora Section Decaloba (Passifloraceae) from Peru. Darwiniana, nueva serie 7(2): 279-288.

Anew species of Passiflora Section Decaloba, P. andicola, endemic in northwest Peru is here described and illustrated. This new herbaceous climber differs from its related species in the indumentum, flower morphology and geographical distribution. Passiflora andicola inhabits montane forests of Cajamarca and Lambayeque Departments. In addition, the morphology and distributional range of $P$. andicola and related species are compared and discussed.
\end{abstract}

Keywords. Endemism; Passiflora subgenus Decaloba; passionflower; Peruvian Andes.

Resumen. Esquerre-Ibañez, B. 2019. Una nueva especie de Passiflora Sección Decaloba (Passifloraceae) de Perú. Darwiniana, nueva serie 7(2): 279-288.

En este trabajo se describe e ilustra una nueva especie de Passiflora Sección Decaloba, $P$. andicola, endémica del noroeste de Perú. Ésta nueva trepadora herbácea difiere de sus especies más afines en el indumento, la morfología floral y la distribución geográfica. Passiflora andicola habita bosques montanos en los Departamentos Cajamarca y Lambayeque. Adicionalmente, se presenta una comparación morfológica y distribucional de $P$. andicola y sus especies afines.

Palabras clave. Andes peruanos; endemismo; flor de la pasión; Passiflora subgénero Decaloba.

\section{INTRODUCCIÓN}

Passiflora L. es el género más numeroso de las Passifloraceae, con más de 500 especies; comprende plantas trepadoras herbáceas o leñosas y de porte arbóreo, de climas tropicales a subtropicales (Ulmer \& MacDougal, 2004; Muschner etal., 2012). Algunas de sus especies poseen importancia comercial por sus frutos comestibles, por su potencial ornamental o por los principios activos que pueden extraerse de ellas (Hernández \& Bernal, 2000; Dhawan et al., 2004); además, revisten importancia ecológica debido a sus interacciones planta-animal (Gilbert, 1982; Dell'Aglio et al., 2016; De Castro et al., 2018).

Para Perú los inventarios y revisiones generales reportan cerca de 90 especies (Zarucchi, 1993; Ulloa Ulloa et al., 2017) y el descubrimiento de nuevos taxones continúa (Esquerre, 2017, 2019); en esta oportunidad, se presenta la descripción de una nueva especie endémica del norte peruano, una con dos poblaciones conocidas hasta el momento en el Departamento de Cajamarca, Distrito Huambos, y la otra en el Departamento de Lambayequem Distrito Kañaris. 


\section{TRATAMIENTO TAXONÓMICO}

Passiflora andicola B. Esquerre, sp. nov. TIPO: Perú, Cajamarca, Chota, Huambos, relicto de bosque, 2300 m s.m., 10-XII-2018, (fl,fr), B. Esquerre 200 (holotipo HUT; isotipo CPUN). Figs. 1-3.

Herbaceous vine, 2-4 $m$, pubescent with curved trichomes 0.1-0.3 mm, stem striate, stipules 3-5 $\times$ 0.4-0.6 mm, linear, falcate. Petiole glandless, $1-4 \mathrm{~cm}$. Lamina 2-3 lobed, 3-7.5 × 2-6 cm, 2-11 conspicuous laminar nectaries. Peduncles 1-4 cm, in pairs or solitary. Bracts linear-lanceolate, scattered, 2-4 $\times$ 0.4-0.6 mm. Flowers 4-4.3 cm in diameter. Sepals oblong-lanceolate 1.7-2 × 0.6-0.8 cm, reddish to greenish abaxially, white adaxially. Petals oblonglanceolate 0.7-1 $\times 0.2-0.3 \mathrm{~cm}$, white. Corona in 2 series, outer series up to $5 \mathrm{~mm}$, liguliform, curved at apex, sometimes dilated, white with 2-3 purple bands, inner series 3-4 mm, white to greenish, flattened 1-2(3) clavate at apex. Androgynophore 10 mm. Ovary 2-3 × 2.5-3.5 mm, globose, pubescent, greenish. Globose berry 1-1.5 × 1-1.5 cm, pubescent, green when unripe, black when ripe; seeds obovoid, black, transversely 4-5 surcate, 3-3.3 × 2-2.5 mm.

Trepadora herbácea, de 2-4 $\mathrm{m}$ de largo, pubescente, con tricomas de 0,1-0,3 mm, curvados, generalmente dispuestos sobre toda la planta. Tallo estriado, flexuoso y verdoso en ramas jóvenes; duro, purpúreo y en zig-zag en ramas maduras. Estípulas lineares, falcadas, $3-5 \times 0,4-0,6 \mathrm{~mm}$, verdosas o púrpuras. Peciolo $1-4 \mathrm{~cm}$, terete, a veces estriado, sin glándulas; lámina 2-3 lobada, 1-7,5 × 2-6 cm, base cordada, obtusa o a veces truncada, margen entero, ápice de lobos redondeados, a veces los lobos son inconspicuos y la lámina parece truncada, raramente el lobo central sobrepasa a los laterales; nervaduras laterales de hasta 7,6 cm; cara adaxial verde en hojas maduras, variegada, con máculas blancas sobre las nervaduras laterales en hojas jóvenes; nectarios laminares 2-11, conspicuos en la sección basal de la lámina foliar y algunos dispersos en la parte central, con ocelos amarillentos a anaranjados; cara abaxial verde en hojas maduras, púrpura en hojas jóvenes, nectarios con borde púrpura, a veces rojizo, y el centro amarillento a verdoso. Pedúnculos en pares o raramente solitarios, $2-4 \mathrm{~cm}$. Brácteas 3, libres, enteras, esparcidas, linear-lanceoladas, rojizas a púrpuras, $2-4 \times 0,4-0,6 \mathrm{~mm}$. Botones florales de hasta $1,4 \times 2,5 \mathrm{~cm}$, campanulados, rojizos. Flores en antesis de 4-4,3 cm de diámetro, horizontales a erectas; hipanto corto, campanulado, de hasta $5 \mathrm{~mm}$ de altura; sépalos 5, oblongolanceolados, ensanchados en la base, rojo oscuro con rebordes blancos a verdosos abaxialmente, blancos adaxialmente, 1,7-2 × 0,6-0,8 cm; pétalos 5, oblongo-lanceolados, blancos, 0,7-1 × 0,2-0,3 $\mathrm{cm}$. Corona filamentosa en 2 series, serie externa 4-5 mm, liguliforme, a veces ligeramente falcada, de ápice curvado a veces dilatado, filamento blanco a crema con algunos matices púrpuras y verdosos, formando en toda la serie dos a tres bandas púrpuras; serie interna de 3-4 mm, filamento blanco a verde, a menudo con máculas y puntuaciones púrpuras, aplanado, con el ápice 1-2(3) clavado, a veces con varias incisiones apicales de manera irregular. Anillo nectarífero púrpura. Opérculo plicado, verdoso, diminutamente denticulado, lobulado, inciso en $1 \mathrm{~mm}$, de margen irregular, púrpura, con máculas púrpuras en la mitad inferior y puntuaciones púrpuras en la mitad superior, de 2,5-3 mm de altura. Limen de $1 \mathrm{~mm}$, denticulado, púrpura, incurvado hacia el androginóforo. Androginóforo de $10 \mathrm{~mm}$ de altura, blanco purpúreo. Filamentos estaminales de 7-8 mm, verdosos, anteras 3-4 × $1 \mathrm{~mm}$, verdosas a púrpuras. Ovario globoso, pubescente, $2-3 \times 2,5$ 3,5 mm, verde; estilos 3 , de 6-7 mm, verdosos con máculas púrpuras o totalmente púrpuras hacia el ápice, estigma verdoso. Baya globosa, verde cuando inmadura, negra al madurar, pubescente, 1-1,5 $\times 1-1,5 \mathrm{~cm}$; semillas 50-55, obovoides, negras, transversalmente $4-5$ surcadas, $3-3,3 \times 2-2,5 \mathrm{~mm}$.

Etimología. El epíteto específico deriva del latín "andicolus" y hace referencia al lugar donde habita esta especie, la cordillera de los Andes.

Distribución y hábitat. La localidad tipo de Passiflora andicola se encuentra en los alrededores de Huambos, Provincia de Chota, Departamento Cajamarca, sobre los $2300 \mathrm{~m}$ s.m. compartiendo hábitat de bosque montano con otras especies como Passiflora cumbalensis (Karst.) Harms, P. manicata (Juss.) Pers., Clusia spp. (Clusiaceae), Polylepis spp. (Rosaceae), Oreocallis grandiflora (Lam.) R. Br. (Proteaceae), Oreopanax spp. (Araliaceae), Dodonaea viscosa Jacq. (Sapindaceae), Myrcianthes spp. (Myrtaceae), Piper spp. (Piperaceae). 


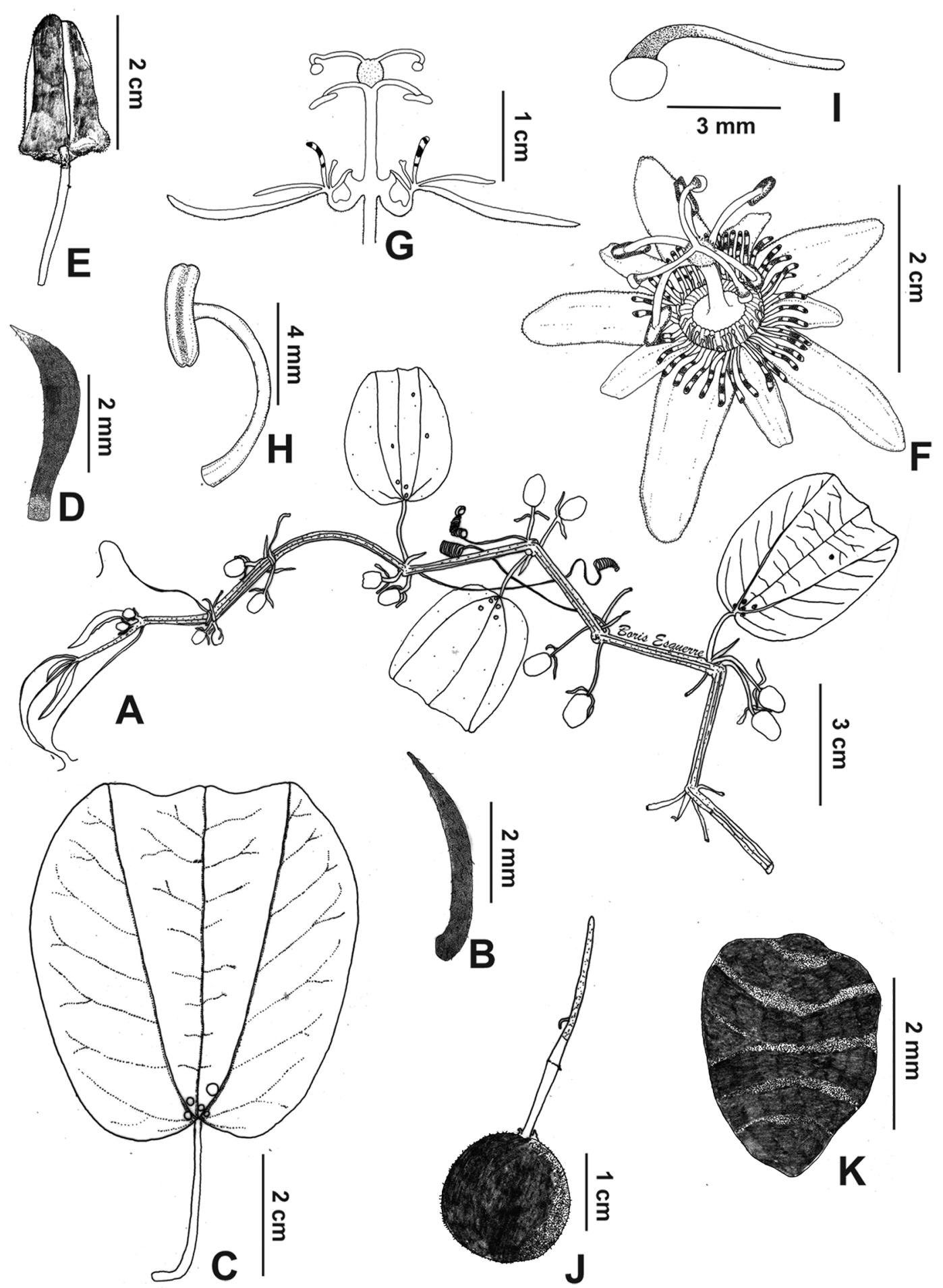

Fig. 1. Passiflora andicola. A, hábito, porción terminal de un tallo. B, estípula. C, hoja. D, bráctea. E, botón floral. F, flor, en antesis. G, flor, corte longitudinal. H, estambre. I, estilo y estigma. J, fruto maduro. K, semilla. De B. Esquerre 200 (HUT). Ilustración de B. Esquerre 


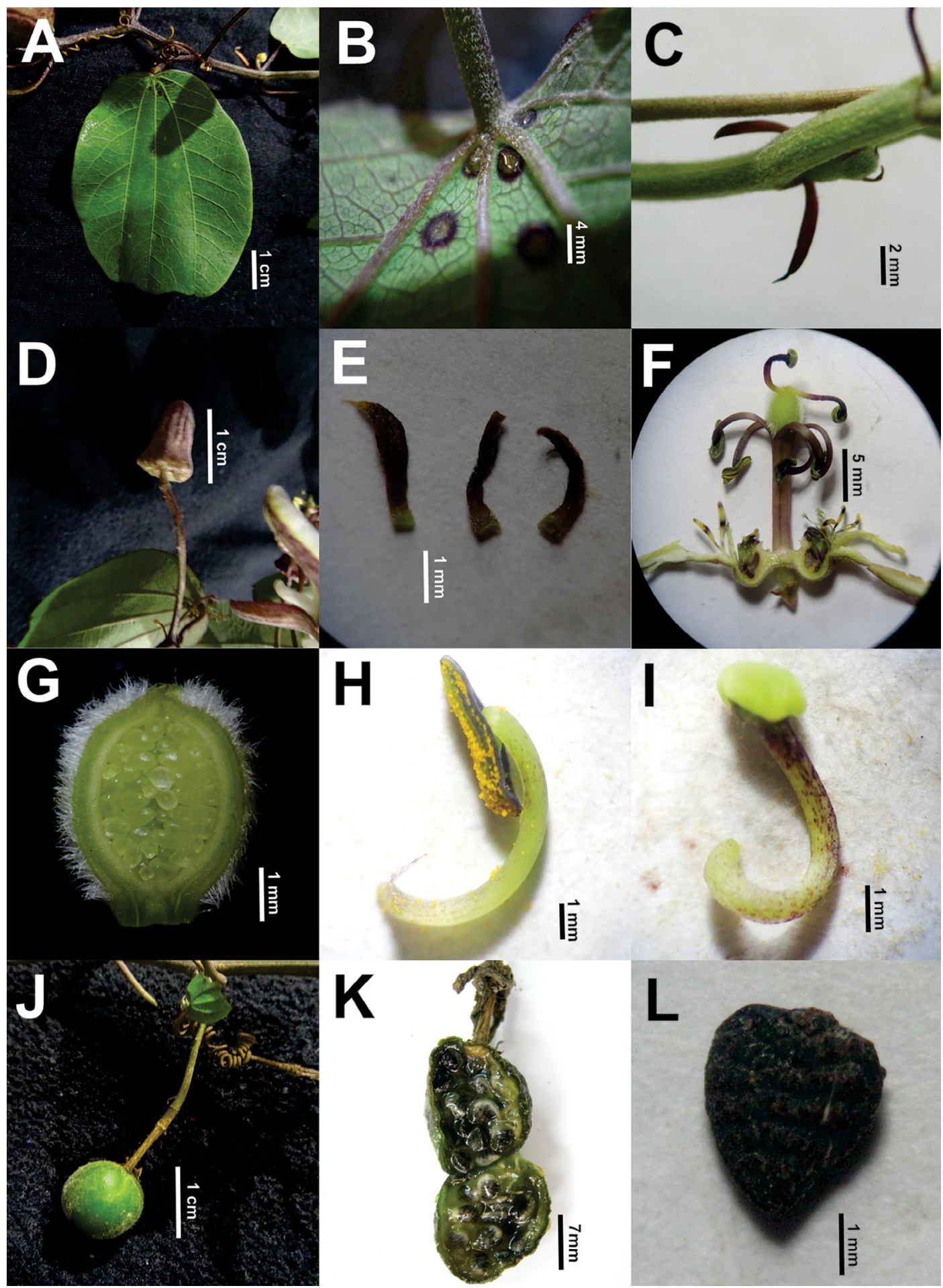

Fig. 2. Passiflora andicola. A, hoja. B, nectarios laminares. C, estípulas. D, botón floral. E, brácteas. F, flor, corte longitudinal. G, ovario, corte longitudinal. H, estambre. I, estilo y estigma. J, fruto inmaduro. K, fruto maduro exhibiendo semillas. L, semilla. Fotografías de B. Esquerre. Figura en color en la versión en línea http://www.ojs.darwin.edu.ar/index.php/darwiniana/article/view/842/1171 

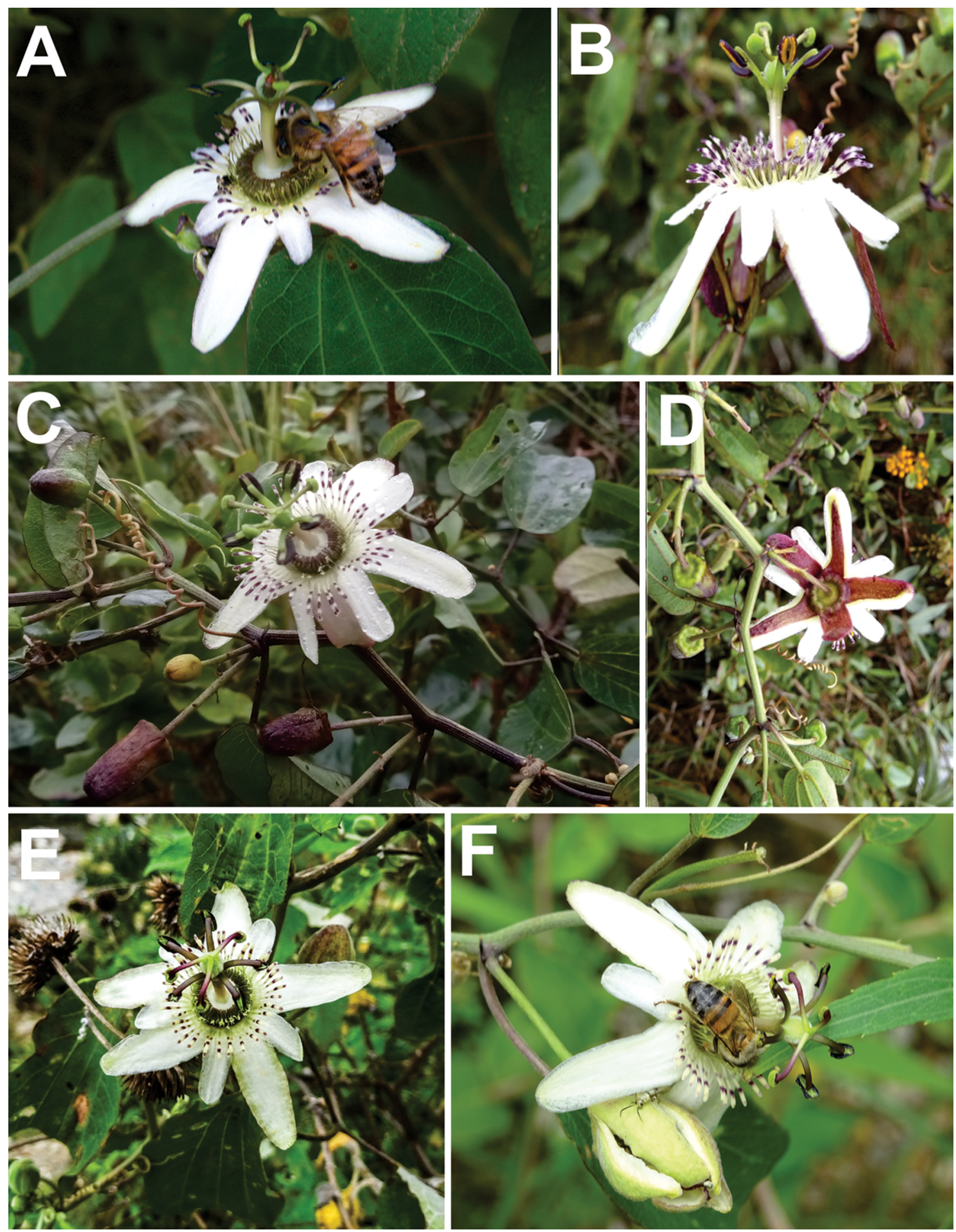

Fig. 3. Passiflora andicola, individuos floríferos in situ. A-D, alrededores de Huambos (Distrito Huambos, Departamento Cajamarca). E-F, alrededores del río Kañariaco (Distrito Kañaris, Departamento Lambayeque). Fotografías de B. Esquerre. Figura en color en la versión en línea http://www.ojs.darwin.edu.ar/index.php/ darwiniana/article/view/842/1171 


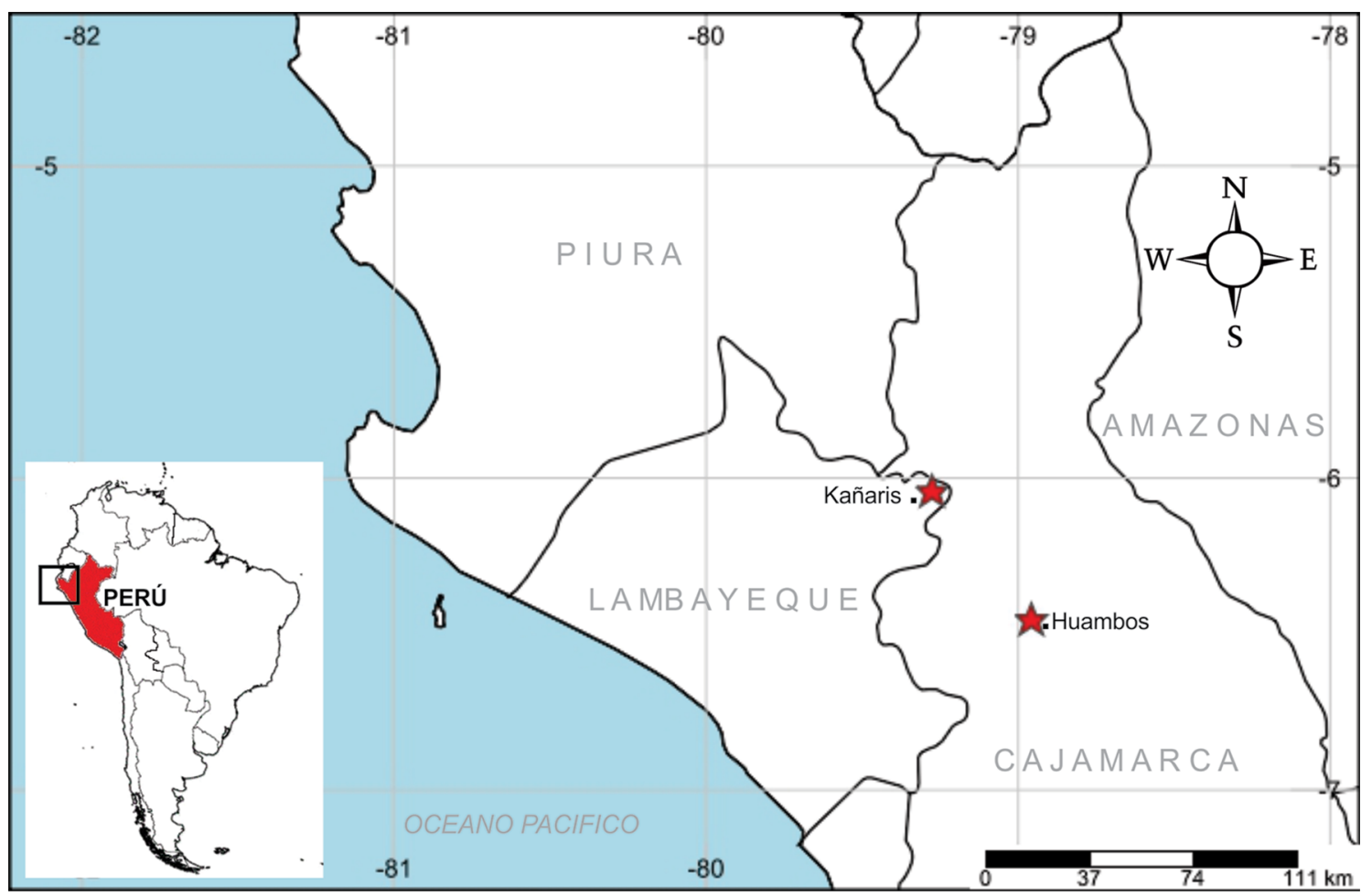

Fig. 4. Distribución geográfica de Passiflora andicola con las dos poblaciones reportadas hasta el presente en el noroeste de Perú.

Una segunda población ha sido hallada a $57 \mathrm{~km}$ al noroeste de Huambos, en el Distrito de Kañaris, Departamento Lambayeque, en un bosque con componentes florísticos semejantes (Fig. 4).

Ecología. Se observó que las flores de Passiflora andicola son visitadas frecuentemente por la "abeja europea" (Apis mellifera L.) tanto en la población de Cajamarca como en la de Lambayeque.

Observaciones. De acuerdo a la clasificación infragenérica actual (Feuillet \& MacDougal, 2004), Passiflora andicola se ubica dentro de Passiflora sección Decaloba DC., por poseer flores y frutos pequeños, opérculo plicado, estilos delgados, semillas transversalmente surcadas, hojas 2-3 lobadas generalmente con el lobo central reducido, nectarios laminares, fruto indehiscente de tipo baya y ápice cernuo.

Durante la revisión de material de herbario se encontró un espécimen colectado en el Departamento de Amazonas, Weigend 98/453 (CPUN, USM, F, $\mathrm{M})$, con características vegetativas similares a las de Passiflora andicola pero, lamentablemente, estéril, de modo que no ha podido determinarse con certeza su identidad. Sería interesante realizar nuevas colectas en esa área a fin de evaluar la posible existencia de una tercera población de Passiflora andicola, ampliándose su distribución a esta región vecina.

Vegetativamente, Passiflora andicola es similar a varias especies de la sección Decaloba, en especial a Passiflora alnifolia Kunth, con hojas 2-3 lobadas, más largas que anchas, pero que puede distinguirse por características florales: la corona externa posee filamentos ligeramente más largos $(0,8 \mathrm{~cm})$ con el tercio superior falcado y el ápice agudo, de color blanco o verdoso y amarillento, las bandas de color son púrpuras a castañas, de 1 a 3 , los pétalos son más largos $(1,4 \mathrm{~cm})$ y el fruto es más grande $(1,9 \mathrm{~cm})$. P. alnifolia habita en la zona andina de Colombia y Ecuador (Tropicos, 2019) donde posee dos formas características por la tonalidad amarillenta o blanquecina de la corona; su presencia en territorio peruano aún no ha sido reportada. 
Tabla 1. Comparación de los caracteres morfológicos más representativos de Passiflora andicola y sus especies afines.

\begin{tabular}{|c|c|c|c|c|}
\hline & P. andicola & P. pascoensis & P. bogotensis & P. alnifolia \\
\hline Distribución & $\begin{array}{l}\text { Perú: Cajamarca, } \\
\text { Lambayeque }\end{array}$ & $\begin{array}{l}\text { Perú: Huánuco, Pasco, } \\
\text { Cusco, San Martín }\end{array}$ & Colombia, Venezuela & Colombia, Ecuador \\
\hline Indumento & pubescente & pubescente & viloso a tomentoso & glabrescente a tomentoso \\
\hline Pedúnculo: longitud & $2-4 \mathrm{~cm}$ & $2-2,8 \mathrm{~cm}$ & $3-4 \mathrm{~cm}$ & $4-6 \mathrm{~cm}$ \\
\hline $\begin{array}{l}\text { Botón floral: } \\
\text { forma y color }\end{array}$ & $\begin{array}{l}\text { campanulado, rojizo a } \\
\text { rojizo-verdoso }\end{array}$ & $\begin{array}{l}\text { campanulado, } \\
\text { blanco-verdoso }\end{array}$ & $\begin{array}{l}\text { campanulado con la } \\
\text { base anular, rojizo a } \\
\text { rojizo-verdoso }\end{array}$ & $\begin{array}{l}\text { campanulado, rojizo a rojizo- } \\
\text { verdoso }\end{array}$ \\
\hline Flor en antesis: diámetro & $4-4,3 \mathrm{~cm}$ & $7-8 \mathrm{~cm}$ & $4 \mathrm{~cm}$ & $4-5 \mathrm{~cm}$ \\
\hline Sépalos: dimensiones & $1,7-2 \times 0,6-0,8 \mathrm{~cm}$ & $2,7-3 \times 1,8-2 \mathrm{~cm}$ & $1-1,5 \times 0,5 \mathrm{~cm}$ & $1-2,4 \times 0,3-0,9 \mathrm{~cm}$ \\
\hline Pétalos: dimensiones & $0,7-1 \times 0,2-0,3 \mathrm{~cm}$ & $2,7-3 \times 1,8-2 \mathrm{~cm}$ & $0,6-0,9 \mathrm{~cm}$ & $0,7-1,4 \times 0,1-0,5 \mathrm{~cm}$ \\
\hline $\begin{array}{l}\text { Corona externa: } \\
\text { longitud del filamento }\end{array}$ & $0,4-0,5 \mathrm{~cm}$ & $1,5 \mathrm{~cm}$ & $0,4-0,5 \mathrm{~cm}$ & $0,4-0,8 \mathrm{~cm}$ \\
\hline \multicolumn{5}{|l|}{ Color del filamento: } \\
\hline Base & blanco & $\begin{array}{l}\text { blanco a verdoso con } 2 \\
\text { bandas púrpuras }\end{array}$ & verdoso o púrpura & blanco o verdoso \\
\hline Centro & $\begin{array}{l}\text { blanco a verdoso- } \\
\text { amarillento con 1-2 } \\
\text { bandas púrpuras }\end{array}$ & $\begin{array}{l}\text { blanco con } 2 \text { bandas } \\
\text { púrpuras }\end{array}$ & verdoso a blanco & $\begin{array}{l}\text { blanco, verdoso o amarillo, con } \\
\text { puntuaciones púrpuras y 1-3 } \\
\text { bandas púrpuras a castaños }\end{array}$ \\
\hline Ápice & $\begin{array}{l}\text { blanco con } 0-1 \text { bandas } \\
\text { púrpuras }\end{array}$ & $\begin{array}{l}\text { blanco con } 2 \text { bandas } \\
\text { púrpuras }\end{array}$ & $\begin{array}{l}\text { amarillo o blanco con } \\
1-2 \text { bandas púrpuras }\end{array}$ & blanco, crema o amarillo \\
\hline $\begin{array}{l}\text { Corona externa: } \\
\text { Forma del ápice }\end{array}$ & $\begin{array}{l}\text { curvado, a veces } \\
\text { dilatado, redondeado }\end{array}$ & $\begin{array}{l}\text { curvado, dilatado, } \\
\text { redondeado }\end{array}$ & $\begin{array}{l}\text { curvado, dilatado, } \\
\text { capitado }\end{array}$ & falcado, dilatado, agudo \\
\hline Estilos: color & verde a purpúreo & $\begin{array}{l}\text { rojizo, purpúreo a } \\
\text { castaño }\end{array}$ & púrpura a castaño & púrpura a castaño \\
\hline Fruto: dimensiones & $1-1,5 \times 1-1,5 \mathrm{~cm}$ & $2,5-3 \times 2,3-2,6 \mathrm{~cm}$ & $1-1,5 \times 1-1,5 \mathrm{~cm}$ & $1,3-2 \times 1-2 \mathrm{~cm}$ \\
\hline Fruto: forma & globoso & elipsoidal & globoso & globoso a ovoide \\
\hline Semilla: dimensiones & $0,3-0,35 \times 0,25 \mathrm{~cm}$ & $0,3 \times 0,2 \mathrm{~cm}$ & $0,3 \times 0,2 \mathrm{~cm}$ & $0,3-0,4 \times 0,2-0,3 \mathrm{~cm}$ \\
\hline Semilla: forma & obovoide, 4-5 surcada & obovoide, 7 surcada & $\begin{array}{l}\text { ovoide a cuneada } \\
\text { obovoide, } 6 \text {-surcada }\end{array}$ & obovoide, 4-6 surcada \\
\hline
\end{tabular}

Passiflora andicola también se asemeja dentro de territorio peruano a Passiflora pascoensis L.K.Escobar, y fuera de éste a $P$. bogotensis Benth. Con Passiflora pascoensis comparten la coloración de sépalos, pétalos y corona, pero el diámetro de la flor en antesis es mayor $(7-8 \mathrm{~cm})$, los sépalos y pétalos son más grandes $(2,7-3 \times$ $1,8-2 \mathrm{~cm}$ ), la corona muestra una serie externa de filamentos más largos $(1,5 \mathrm{~cm})$ y con más bandas de color purpúreo (5-6); las flores son péndulas y las hojas más grandes $(9,5-14,8 \times 6,5-8,6 \mathrm{~cm})$, el fruto es elipsoidal y más grande $(2,5-3 \times 2,3-2,6$ $\mathrm{cm})$. P. pascoensis habita la selva central y sur de Perú, en los Departamentos de Huánuco, Pasco, Cusco y posiblemente San Martín (Tropicos, 2019); no ha sido reportada más al norte ni al oeste. Con Passiflora bogotensis comparten la forma de las hojas, la coloración de sépalos, pétalos y corona, pero se diferencia principalmente por el botón floral, muy ensanchado en la base, dando la apariencia de un reborde o anillo; también por el indumento, densamente viloso-tomentoso, por los filamentos coronarios con 1-2 bandas de color púrpura, a menudo fusionadas. $P$. bogotensis crece en la zona andina de Colombia y parte de Venezuela, no llega a Perú (Tropicos, 2019).

Las semejanzas y diferencias antes mencionadas, entre $P$. andicola, $P$. alnifolia, $P$. pascoensis y $P$. bogotensis se detallan en la Tabla 1; las flores de cada una de estas especies se ilustran en la Figura 5. 

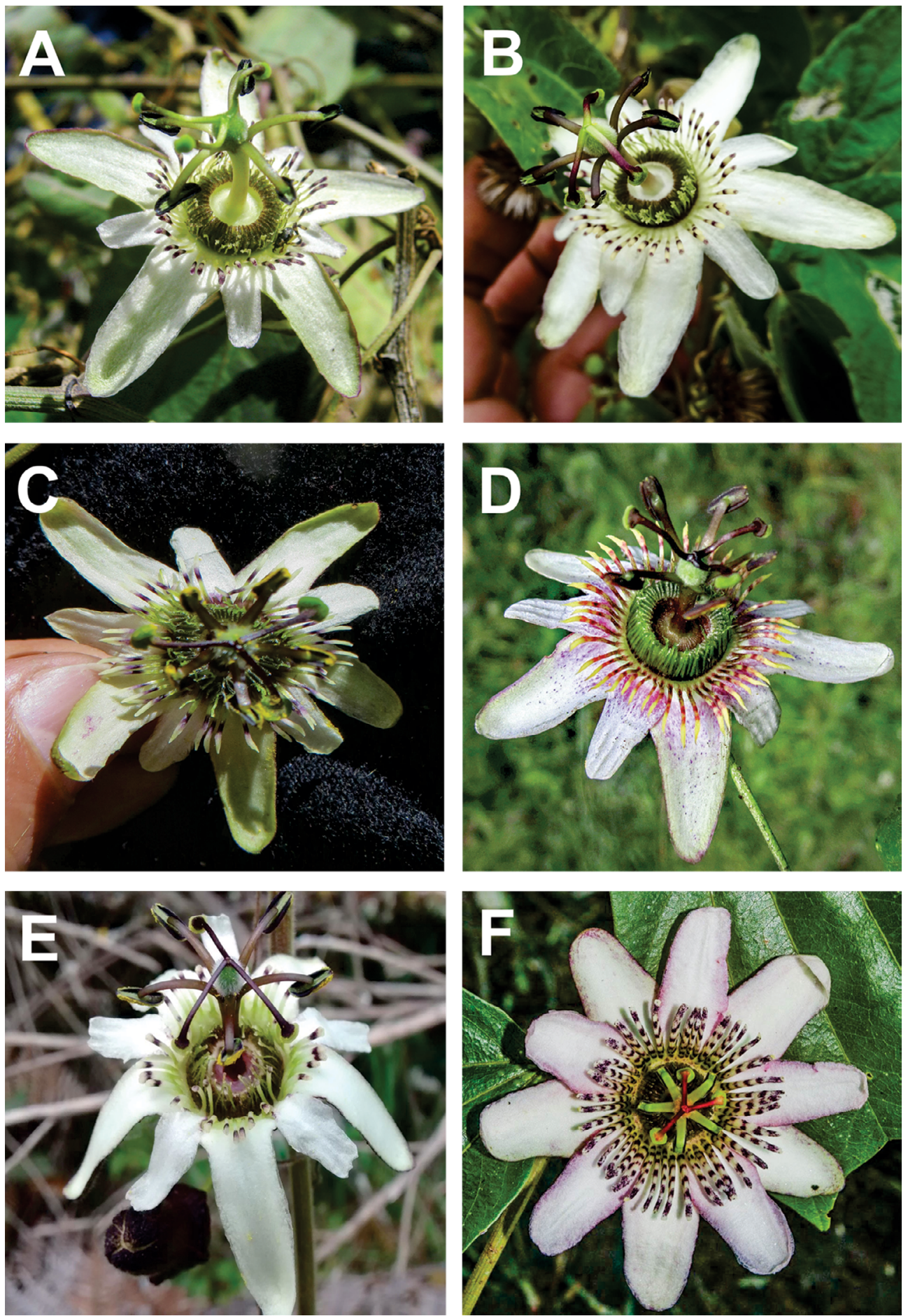

Fig. 5. Passiflora andicola y especies afines, flores en antesis. A, $P$. andicola (Huambos, Cajamarca, Perú). B, P. andicola (Kañaris, Lambayeque, Perú). C, P. alnifolia (Calacalí, Pichincha, Ecuador). D, P. alnifolia (Santa Elena, Antioquia, Colombia). E, P. bogotensis (Chía, Cundinamarca, Colombia). F, P. pascoensis (Oxapampa, Pasco, Perú). Fotografías: Boris Esquerre (A-B), Carlos Cerón (C), Jorge Restrepo (D), Hernán Darío (E), Rodolfo Vásquez-Missouri Botanical Garden (F). Figura en color en la versión en línea http://www.ojs.darwin.edu.ar/index.php/darwiniana/article/view/842/1171 
Grado de conservación. Durante las visitas a la localidad tipo y sus alrededores se pudo constatar el significativo avance urbano y agrícola, tanto en las afueras del pueblo de Huambos como en toda el área de montaña que atraviesa el distrito. En algunos sectores, la cobertura de bosque nativo es muy escasa o nula, habiendo sido reemplazada por especies forestales de Pinus y Eucalyptus, y por cultivos alimenticios. Por su lado, la población de Lambayeque fue hallada en un área de bosque abierto, que poco a poco está siendo devastada por la práctica de la agricultura migratoria y los incendios forestales. Por estos motivos, se propone la categoría de conservación "En Peligro", EN B1ab (iii), de acuerdo a los criterios de la Unión Internacional para la Conservación de la Naturaleza (IUCN, 2001).

\section{Material representativo examinado (Paratipos)}

PERÚ. Cajamarca. Prov. Chota. Distrito Huambos. alrededores de Huambos, 2341 m s. m., 10-XII-2018, (fl), B. Esquerre 201 (HUT, CPUN); 2354 m s. m., 10XII-2018, (fl), B. Esquerre 202 (HUT, CPUN); $2330 \mathrm{~m}$ s. m., 10-XII-2018, (fl), B. Esquerre 203 (HUT, CPUN); 2340 m s. m., 12-II-2019, (fl), B. Esquerre 213 (HUT, CPUN); 2345 m s. m., 12-II-2019, (fl), B. Esquerre 214 (HUT, CPUN); 2340 m s. m., 12-II-2019, (fl), $B$. Esquerre 215 (HUT, CPUN). Lambayeque. Prov. Ferreñafe. Distrito Kañaris, cuenca del Río Kañariaco, ruta de Kañaris a Yoyoca, 2205 m s. m., 14-I-2019, (fl), B. Esquerre 210 (HUT, CPUN); 2210 m s. m., 14-I2019, (fl), B. Esquerre 211 (HUT, CPUN); $2215 \mathrm{~m} \mathrm{~s}$. m., 14-I-2019, (fl), B. Esquerre 212 (HUT, CPUN).

\section{AGRADECIMIENTOS}

Mi gratitud a la Universidad Nacional Pedro Ruiz Gallo, Consuelo Rojas y Leopoldo Vásquez (Perú) por facilitar la movilidad a los sitios de colecta, a Guillermo Delgado (Perú) por permitir el uso de los equipos y ambiente del Laboratorio General de Biotecnología-UNPRG para la disección del material. A John MacDougal (Estados Unidos de América) y Yero Kuethe (Inglaterra) por la revisión del manuscrito y críticas. A Carlos Cerón (Ecuador), Jorge Restrepo, Hernán Darío (Colombia) y Rodolfo Vásquez (Perú) por las fotografías de las especies afines. A Robert Vogt y Tilo Henning (Alemania) por su valiosa ayuda en las consultas al Herbarium Berolinense (Berlin-Dahlem).
A los revisores anónimos y editor por sus aportes en la publicación del presente trabajo. Las colectas botánicas están autorizadas en Perú por el Servicio Forestal y de Fauna Silvestre mediante Resolución $N^{\circ}$ 430-2017-SERFOR/DGGSPFFS.

\section{BIBLIOGRAFÍA}

Dhawan, K, S. Dhawan \& A. Sharma. 2004. Passiflora: a review update. Journal of Ethnopharmacology 94: 1-23. DOI: 10.1016/j.jep.2004.02.023

De Castro, E; M. Zagrobelny; M. Cardoso \& S. Bak. 2018. The arms race between heliconiinae butterflies and Passiflora plants - new insights on an ancient subject. Biological Reviews 93: 555-573. DOI: 10.1111/ brv. 12357

Dell'Aglio, D; M. Losada \& C. Jiggins. 2016. Butterfly learning and the diversification of plant leaf shape. Frontiers in Ecology and Evolution 4: 1-7. DOI: 10.3389/fevo.2016.00081

Esquerre-Ibañez, B. 2017. A new species of Passiflora section Granadillastrum (Passifloraceae) from Jaén, Peru. Phytotaxa 298 (1): 89-95. DOI: 10.11646/ phytotaxa.298.1.10

Esquerre-Ibañez, B. 2019. Una especie nueva ovoimitadora en Passiflora (Passifloraceae) de la Provincia Huancabamba, Piura, Perú. Revista Peruana de Biología 26 (1): 3-8. DOI: 10.15381/rpb.v26i1.15902

Feuillet, C. \& J. M. MacDougal. 2004. A new infrageneric classification of Passiflora. Passiflora 13 (2): 34-35, 37-38.

Gilbert, L. 1982. The coevolution of a butterfly and a vine. Scientific American 247: 110-121. DOI: 10.1038/ scientificamerican0882-110

Hernández, A. \& R. Bernal. 2000. Lista de Especies de Passifloraceae de Colombia. Biota Colombiana 1 (3): 320-335.

IUCN 2001. IUCN Red List Categories and Criteria. Version 3.1. Second Edition. Gland, Switzerland and Cambridge; IUCN, 32 pp. Disponible en: http://www. iucnredlist.org/

Muschner, V. ; P. Zamberlan; S. Bonatto \& L. Freitas. 2012. Philogeny, biogeography and divergence times in Passiflora (Passifloraceae). Genetics and Molecular Biology 35 (4): 1036-1043. DOI: 10.1590/S141547572012000600019

Tropicos.org. Missouri Botanical Garden. 2019, http:// www.tropicos.org 
DARWINIANA, nueva serie 7(2): 279-288. 2019

Ulloa Ulloa, C.; P. Acevedo-Rodríguez; S. G. Beck; M. J. Belgrano; R. Bernal; P. E. Berry; L. Brako; M. Celis; G. Davidse; S. R. Gradstein; O. Hokche; B. León; S. LeónYánez; R. E. Magill; D. A. Neill; M. H. Nee; P. H. Raven; H. Stimmel; M. T. Strong; J. L. Villaseñor Ríos; J. L. Zarucchi; F. O. Zuloaga \& P. M. Jørgensen. 2017. An integrated assessment of vascular plants species of the Americas. Science 358: 1614-1617, f. 1-4. DOI: 10.1126/science.aao0398
Ulmer, T. \& J. MacDougal. 2004. Passiflora: Passionflowers of the world. Timber Press, Portland, Cambridge, $430 \mathrm{pp}$

Zarucchi J. L. 1993. Passifloraceae, en L. Brako \& J.L. Zarucchi (eds.), Catálogo de las Angiospermas y Gimnospermas del Perú. Monographs in Systematic Botany from the Missouri Botanical Garden 45: 876-882. 